\title{
Ethnicity, Spatial Segregation and Length of Stay in Emergency Medicine Departments: The Case of Israel
}

\author{
Keren Semyonov-Tal ${ }^{1}$ \\ Tel-Aviv University, Israel
}

\begin{abstract}
The present paper examines the sources of disparities between Arabs and Jews and between residents of the periphery and residents of the urban center in the length of stay (LOS) in emergency departments (ED) in Israeli hospitals. Israel is a society where all residents are covered by National Health Insurance and have equal access to emergency medicine; thus, no disparities in emergency treatment are expected. Data were obtained from the 2015 "Survey of Satisfaction with Hospitalization in Emergency Medicine Departments" in Israel. The analysis reveals that LOS is considerably shorter for Arabs than for Jews and among residents of the periphery than residents of the urban center. After considering patterns of residential segregation (as well as socio-demographic attributes and indicators of the medical condition) and controlling for perceived waiting time (triage), the LOS disparity between Arabs and Jews vanishes. The findings lead to the conclusion that disparities in LOS in Israeli EDs do not stem from differential treatment by the medical staff but can be fully attributed to patterns of spatial segregation. Differential allocation of medical resources by the state to urban-center regions, compared to peripheral regions, seems to be the source of LOS disparities in emergency departments in Israeli hospitals.
\end{abstract}

Keywords: emergency medicine, ethnic inequalities, health disparities, residential segregation.

Healthcare legislation implemented in many nations, coupled with the ethical codes subscribed to by the medical profession, lead us to the expectation of no disparities between populations concerning emergency medical treatment. Curiously, however, multiple studies exploring emergency medical treatment, especially those on differences by race in the United States, have identified significant disparities in treatment along racial and ethnic lines, with members of ethnic or racial minorities less likely to receive the same quality of emergency treatment as other groups (for studies and discussion in the United States see, for example, Blanchard et al., 2003; Dehon et al., 2017; Richardson et al., 2003). These disparities can be understood in light of the uniqueness of the American medical system. They may not apply to other welfare societies (such as Israel), in which the public healthcare system allows equal access to medical services in general, and emergency medical treatment in particular, to all residents. This paper contributes to the literature on health inequality by examining the sources of disparities in length of stay (LOS) in emergency medicine units between Arabs and Jews in Israeli hospitals.

\footnotetext{
${ }^{1}$ Corresponding Author E-Mail: keren@st-law.co.il
} 


\section{The Conceptual Framework and Previous Research}

Access to health and medical services is viewed in contemporary societies as a fundamental social right for all residents, regardless of socio-economic status or race and ethnicity. That is to say, in welfare states, the government is expected to ensure the provision of adequate healthcare services to all segments of the population, regardless of socio-economic status or ethnic origin. The Ottawa Declaration, approved by the World Health Organization (Alma Ata Declaration, 1978), set the goal of eliminating gaps in access to healthcare. In the spirit of the Ottawa declaration, many nations have begun to implement policies aimed at minimizing, if not totally eliminating, health inequality. In reality, however, most nations are far from achieving equality, either in access to health services or in the attainment of good health across all population groups (e.g., Fiscella et al., 2000; Mackenbach et al., 2008; Semyonov et al., 2013; Williams \& Collins, 1995). More specifically, studies clearly indicate that people belonging to disadvantaged groups (e.g., poor or subordinate ethnic minorities) are disadvantaged in receiving health services equal in quality to those belonging to society's upper strata.

In the case of ethnic and racial minorities, researchers suggest that, in addition to the limitations that derive from the lack of economic resources, racism and discrimination may play a role in producing and perpetuating ethnicity-linked health inequality (for a review, see Williams \& Collins, 1995; see also Armstrong, 2019; Harris et al., 2018; James, 2019; Nazroo 2003; Williams $\&$ Sternthal, 2010). Whereas some portion of these ethnicity-linked health disparities can be attributed to socio-economic gaps, one cannot dismiss the possibility that part of the disparity results from prejudice and discrimination (e.g., Carter et al., 2017; Nazroo, 2003). That is, stereotypes might unwittingly lead to undesired bias and differential treatment (Chapman et al., 2013). Likewise, it is also possible that due to language and cultural barriers, ethnic minorities (immigrants in the main) experience difficulties in emergency departments due to their limited knowledge of the domestic language. Language limitations decrease the ability to communicate effectively, report health problems, fill out forms, and understand guidelines and instructions, leading to increased waiting time in emergency departments (e.g., Bowen, 2001; Flores, 2006; Meuter et al., 2015; Padela \& Punekar, 2009).

Another primary explanation for the disadvantages that ethnic minorities experience in obtaining quality medical services in US hospitals is based on differential insurance coverage rates (e.g., Ku \& Matani, 2001; Zuvekas \& Taliaferro, 2003). Students of ethnic inequalities have uniformly observed that ethnic and racial minorities and poor people, compared to members of the majority population and rich people, are less likely to purchase health insurance (e.g., Monheit \& Vistnes, 2000; Semyonov et al., 2011). The lack of health insurance (or limited insurance coverage) is likely to lead to the overuse of emergency medical treatment as an alternative to conventionalstandard (and expensive) procedures. For people without health insurance, emergency medicine has become an alternative route for obtaining medical services in the United States.

In addition to the impact of prejudice and discrimination and the differential availability of health insurance, several researchers have underscored the role that residential segregation plays in producing racial disparities in access to health services in general and emergency medicine in particular (e.g., Feikin et al., 2009; Guagliardo, 2004; LaVeist, 2005; Morrill et al., 1970; Riley, 2018; Williams \& Collins, 2016). Students of health inequality have repeatedly argued that spatial location within a city and distance from the urban center negatively affects access to quality hospitals and medical services. On this issue, LaVeist (2005), for example, attributed part of the poorer health of blacks in the United States to the limited availability of quality hospitals and clinics in Black neighborhoods. The limited availability of quality medical services in poor and ethnic minority communities' results in the deterioration of health and a decline in the quality of 
emergency services. In line with this logic, Pines et al. (2009) identified, for example, longer waiting times in intensive care units of hospitals with a high proportion of Black patients.

The present paper attempts to contribute to the literature on ethnic inequality in access to health care by focusing on differential treatment of Arabs and Jews in emergency departments in Israel. Specifically, the paper examines first, whether Arabs' LOS, as members of the subordinate ethnic group, is shorter than the LOS of Jews, and second, whether disparities in LOS can be attributed to spatial location or to differential treatment. By so doing the analysis underscore sources for disparities in access to health services. Hence, contributes to a better understanding of patterns and sources of ethnic inequality.

\section{Previous Studies on Inequality in Emergency Medicine}

One of the most used measured indicators that researchers have adopted to capture healthcare service quality disparities is the length of stay (LOS) in the emergency department. LOS pertains to the time that a patient spends from arrival in the ED till release or transfer to an Inpatient department. Researchers, however, do not uniformly agree on the implications of LOS for the quality of medical care. Several researchers suggest that short LOS reflects ED hospital efficiency (e.g., Chaou et al., 2017; Pines et al., 2009); others argue that it reflects the poor quality of treatment (e.g., Thomas et al., 1997). According to Pines et al. (2009) and Chaou et al. (2017), extended hospitalization results in the overuse of resources and overcrowding, which, in turn, leads to a deterioration in the quality of medical treatment available. Following this line of reasoning, several researchers have observed that prolonged LOS in ED is directly associated with patient dissatisfaction, increased medical errors, poorer health outcomes, and higher mortality rates (e.g., Herring et al., 2009; Miro et al., 1999; Moskop et al., 2009; Mowery, 2011; Sprivulis et al., 2006). By way of contrast, Thomas (1997) argued that short LOS might result from premature discharge and may reflect the poor quality of ED treatment.

LOS is the sum of three distinct and mutually exclusive components representing three stages of hospitalization: input, throughput, and output (e.g., Asplin et al., 2003; Hoot \& Aronsky, 2008). The three respective components of ED LOS are: waiting time before initial assessment by a physician or nurse (triage), net time devoted to medical treatment, and waiting time for tests and laboratory results before final discharge. Therefore, when using LOS as an outcome indicator, it is important to adjust the measure for waiting time (triage) in ED before medical treatment and also to focus on the net time devoted to medical treatment as an indicator of the quality of medical treatment (Kyriacou et al., 1999). Curiously, however, very few studies to date have examined "net time devoted to medical treatment" as a measure of LOS in emergency units.

\section{The Israeli Context}

Israel is a multiethnic society inhabited by Jewish immigrants and their offspring (the majority population) and Arabs (the minority group population). Arabs, who have uniformly lived in the region for generations, constitute about $20 \%$ of Israel's population. Despite considerable ethnic variations within the Jewish population, the most salient and meaningful ethnic split in Israeli society is between Jews and Arabs. More specifically, Israeli Arabs are disadvantaged, relative to Jews, in every aspect of socio-economic status, including education, occupation, earnings, wealth, political power, and standard of living (e.g., Lewin-Epstein \& Semyonov 1993), as well as in health status and life expectancy (e.g., Baker, F. A. et al., 2020; Daoud et al., 2018; 
Hatab, 2011; Manor et al., 2000; Saabneh, 2015; Shuval, 1990). Arabs are also heavily concentrated in the country's peripheral regions, where employment opportunities, cultural centers, and medical facilities are relatively scarce. More specifically, most Arabs in Israel reside in homogeneous all-Arab villages and small towns in the peripheral regions of the country, at a distance from major medical facilities. By way of contrast, Israel's Jewish population is largely concentrated in the major cities and urban communities in the country's central regions (e.g., Lewin-Epstein \& Semyonov, 1993).

The health care system in Israel is publicly funded, and national health insurance covers all Israeli residents regardless of ethnicity or socio-economic status. Health services are provided via a series of general public hospitals and an elaborate network of clinics, pharmacies, and preventive medical services scattered across the country. The National Health Law guarantees a defined and equal "basket" of health services for all Israeli citizens. The inclusion of all citizens under the rule of law aims to ensure that all patients have equal access to hospitals and healthcare facilities.

Whereas primary care facilities are widespread and accessible in most Israeli communities, the major hospitals are not equally distributed across the country (e.g., Shuval, 1990). According to an Israel Ministry of Health (2016) report, the major hospitals with advanced medical equipment and facilities are located in the country's central regions. Currently, of the 33 general hospitals in Israel, the majority (17) are located in the central region around Tel Aviv and its environs and the other urban centers (e.g., Haifa and Jerusalem). Relatively very few major medical facilities are located in the peripheral regions of the country.

Studies in Israel (and other countries) suggest that distance from an urban-center region negatively affects access to quality hospitals and medical services and may lead to disparities in health between residents of urban centers and residents of small peripheral communities (e.g., Lubetzky et al., 2011; Ministry of Health, 2016; Nirel et al., 2000; Shuval, 1990). Disparities in the provision of health services across the country are clearly evident in Israel. For example, between 1995 and 2008, the ratio of medical doctors for every 1000 citizens was 2.5 in the peripheral districts compared to 3.7 in the urban-center. Similar gaps were reported regarding nurses and beds for hospitalization per 1000 of the population (Israel Ministry of Health, 2010). Likewise, the ratio of physicians to patients in the central districts of the country is more than twice that of physicians employed in the northern districts (5.1 physicians versus 2.3 physicians per thousand patients) and almost twice as much as in the southern district (3.1 physicians per thousand patients) (See also Lubetzky et al., 2011). Such spatial disparities in resource allocation may have significant consequences for disparities between sub-populations concerning access to medical services in general and access to emergency medicine in particular. Therefore, in light of the ethnic split and spatial disparities in Israeli society, the analysis that follows focuses on emergency medicine LOS disparities between Jews and Arabs and between residents of the urban center and residents of the periphery.

\section{Data Source and Variables}

Data for the analysis were obtained from the 2015 "Survey of satisfaction with Hospitalization in Emergency Medicine Departments" conducted by the Israeli Ministry of Health. The population included in the survey is composed of patients who visited emergency departments in Israeli hospitals, excluding psychiatric care centers, gynecology departments, and pediatric departments. To ensure that the survey findings accurately represent patients in all hospitals, a weighting procedure was applied to reflect the actual number of patients in each of the emergency departments in 26 hospitals in Israel. Questionnaires were administered up to 14 days after discharge from the emergency department. Interviews were conducted in the patient's preferred 
language. A total of 10,152 patients were interviewed (representing a 59\% response rate). The survey was designed by the Israeli Ministry of Health in accordance with patient experience surveys conducted around the world (such as- England, Australia and in OECD countries). The survey questionnaire underwent a validation process: in the first phase, face-to-face interviews were conducted with patients in the wards and in the second phase, a telephone survey. Based on the results of the validation, the final questionnaire was formulated. The interviews were conducted as part of a telephone survey during the months of May-August 2015. The analysis was limited to the 9803 patients who provided complete information.

The survey included detailed information about the patients' socio-demographic characteristics, medical condition, hospitalization experience in the ED, and length of time spent in the ED. Ethnic origin and region of residence were used as the two main predictors of LOS. Ethnicity was defined by the distinction between Jews and Arabs (Jews = 1), and the region was based on the distinction between the urban center $(=1)$ and communities in the periphery. In addition to the two predictors of LOS, three socio-demographic attributes of patients were used as control variables, namely gender $($ men $=1)$, age (in years), and education (academic $=1$ ).

In addition to the socio-demographic attributes of patients, a series of questions pertaining to the patient's medical condition and type of medical problem were available in the data set. The complexity or severity of the problem was defined as a dummy variable distinguishing between urgent $\mathrm{ED}(=1)$ and unurgent $(=0)$; the type of medical problem was defined by a set of 18 dummy variables, each representing a distinct medical category ${ }^{2}$. Patients also reported perceived waiting time (triage for nurses and doctors). Triage was defined along four possible categories (no, short, medium, or long) and was introduced to the analysis as a set of dummy variables. Perceived triage levels for doctors and nurses were converted to minutes (no $=0 \mathrm{~min}$; short $=5 \mathrm{~min}$; medium $=10$ $\mathrm{min}$; long= $15 \mathrm{~min})^{3}$.

Two measures for the length of stay in the ED (as indicators of quality of care) were used as the dependent variables in the analysis. The first was TOTLOS (total duration of stay in the ED, in minutes, from entry to discharge); the second was NETLOS (TOTLOS - (triage doctors + triage nurses)), which pertains to time devoted to medical treatment minus waiting time for medical staff. The list of variables, their detailed definitions, and measurements are provided in Appendix Table A.

\section{Data Analysis and Results}

\section{Descriptive Overview}

Table 1 presents and compares the mean values for TOTLOS and NETLOS (as well as medical conditions, perceived triage, and the socio-demographic characteristics of the population) of Jews and Arabs (Columns 1 and 2) and patients treated in the urban center and the peripheral regions of the country (Column 3 and 4), for a descriptive overview. The distribution of the 18 types of medical problems for which patients were treated in the ED is listed in Appendix B.

\footnotetext{
218 dummy variables were introduced to the analysis to capture variations in the type of medical problem experienced by the patient. (The list is provided in the Appendix Table B)

3 In accordance with Ministry of Health guidelines in Israel (e.g., Guideline \# 2/2015), triage of a patient by a staff member shall not exceed 15 minutes. Accordingly, a perceived short waiting time for medical personal was set at 5 minutes; 10 minutes for a medium time; and 15 minutes for a long waiting time.
} 
Table 1

Mean (s.d.) or Percentage for the Variables Included in the Analysis by the Ethnic Origin of the Patient/Location

\begin{tabular}{|c|c|c|c|c|c|}
\hline Variable & & Arab & Jew & Periphery & Center \\
\hline TOTAL LOS & In minutes & $\begin{array}{l}238.89 \\
(319.6)\end{array}$ & $\begin{array}{l}262.13 \\
(330.0)\end{array}$ & $\begin{array}{c}188.15 \\
(207.09)\end{array}$ & $\begin{array}{c}292.9 \\
(370.68)\end{array}$ \\
\hline NET LOS & In minutes & $\begin{array}{c}219.32 \\
(406.99)\end{array}$ & $\begin{array}{c}243.58 \\
(329.48)\end{array}$ & $\begin{array}{c}169.32 \\
(206.65)\end{array}$ & $\begin{array}{c}274.28 \\
(370.22)\end{array}$ \\
\hline Gender \% & $\begin{array}{l}\text { Female } \\
\text { Male }\end{array}$ & $\begin{array}{l}37.7 \\
62.3\end{array}$ & $\begin{array}{l}49.3 \\
50.7\end{array}$ & $\begin{array}{l}45 \\
55\end{array}$ & $\begin{array}{l}48.6 \\
51.4\end{array}$ \\
\hline Education \% & $\begin{array}{l}\text { Non- } \\
\text { academic } \\
\text { Academic }\end{array}$ & 75.9 & 63.6 & 71 & 63.6 \\
\hline Location \% & $\begin{array}{l}\text { Peripheral } \\
\text { Center }\end{array}$ & $\begin{array}{l}57.3 \\
42.7\end{array}$ & $\begin{array}{l}26.5 \\
73.5\end{array}$ & ---- & ---- \\
\hline Age & & $\begin{array}{c}29.9 \\
(22.98)\end{array}$ & $\begin{array}{l}37.32 \\
(27.0)\end{array}$ & $\begin{array}{c}32.49 \\
(25.15)\end{array}$ & $\begin{array}{c}38.09 \\
(27.18)\end{array}$ \\
\hline Ethnicity \% & $\begin{array}{l}\text { Arab patients } \\
\text { Jewish } \\
\text { patients }\end{array}$ & ---- & ---- & $\begin{array}{l}31.9 \\
68.1\end{array}$ & $\begin{array}{l}11.2 \\
88.8\end{array}$ \\
\hline $\begin{array}{l}\text { Complexity of } \\
\text { hospitalization } \%\end{array}$ & $\begin{array}{l}\text { Regular ED } \\
\text { Urgent ED }\end{array}$ & $\begin{array}{c}5.3 \\
94.7\end{array}$ & $\begin{array}{c}5.2 \\
94.8\end{array}$ & $\begin{array}{c}8.6 \\
91.4\end{array}$ & $\begin{array}{c}3.6 \\
96.4\end{array}$ \\
\hline Triage nurses $\%$ & $\begin{array}{l}\text { No } \\
\text { Short } \\
\text { Medium } \\
\text { Long }\end{array}$ & $\begin{array}{c}3 \\
31.4 \\
41.2 \\
24.3\end{array}$ & $\begin{array}{c}6.5 \\
37.4 \\
37 \\
19.1\end{array}$ & $\begin{array}{c}5.6 \\
34.9 \\
38.5 \\
29.9\end{array}$ & $\begin{array}{c}6.1 \\
37.3 \\
37.2 \\
19.5\end{array}$ \\
\hline Triage doctors $\%$ & $\begin{array}{l}\text { No } \\
\text { Short } \\
\text { Medium } \\
\text { Long }\end{array}$ & $\begin{array}{c}1.7 \\
25.7 \\
42 \\
30.6\end{array}$ & $\begin{array}{c}3.3 \\
25.1 \\
37.5 \\
34.1\end{array}$ & $\begin{array}{c}2.6 \\
25.7 \\
39 \\
32.7\end{array}$ & $\begin{array}{c}3.1 \\
25.1 \\
38 \\
33.8\end{array}$ \\
\hline Triage nurse & $X$ minutes & $\begin{array}{c}9.34 \\
(4.06)\end{array}$ & $\begin{array}{c}8.43 \\
(4.26)\end{array}$ & $\begin{array}{c}8.74 \\
(4.24)\end{array}$ & $\begin{array}{c}8.5 \\
(4.24)\end{array}$ \\
\hline Triage doctor & $X$ minutes & $\begin{array}{l}10.07 \\
(3.97) \\
\end{array}$ & $\begin{array}{l}10.12 \\
(4.25) \\
\end{array}$ & $\begin{array}{l}10.09 \\
(4.14) \\
\end{array}$ & $\begin{array}{l}10.12 \\
(4.22) \\
\end{array}$ \\
\hline $\mathrm{N}=9803 / 10,152$ & & 1746 & 8057 & 3215 & 6937 \\
\hline
\end{tabular}

The data in Columns 1 and 2 reveal meaningful differences between the two ethnic groups concerning the length of stay in an emergency unit. The duration of stay (TOTLOS) in the ED for Jews was longer, by 24 minutes (on average), than for Arab patients. Likewise, the average net time devoted to medical treatment (NETLOS) in the emergency unit was longer for Jews than for Arabs (by 24 minutes). Curiously, the ethnic disparity in NETLOS is virtually identical to the ethnic gap in TOTLOS. The identical ethnic disparities in the two measured indicators of the length of stay seemingly reflect the absence of systematic ethnic differences in perceived waiting times for medical personal.

The data do not reveal any ethnic differences in the complexity of the medical problem reported (the medical situation of $95 \%$ of the patients surveyed, whether Jews or Arabs, was defined 
as "urgent"). Nevertheless, some meaningful differences are present in the type of medical problem that Arabs and Jews experienced (data presented in Appendix B). Whereas Arabs were more likely to be treated by orthopedists, pediatricians, and internal medicine physicians, Jews were more likely to be treated by surgeons, neurologists, eyes specialists, and gynecologists. In addition, the data show some meaningful differences in the socio-demographic attributes of Jewish and Arab patients, with Arabs being younger, less likely to hold an academic degree, and more likely to be males. The most striking ethnic difference, however, regards the spatial location of the hospital. Arabs, who are concentrated in the peripheral regions of the country, are more likely to seek treatment in hospitals located in the periphery (nearly 60\% of Arabs, as compared to $26.5 \%$ of Jews).

The differences in length of stay between patients in the urban center and patients in the periphery (Columns 3 and 4) are more striking than the ethnic disparities (as detailed previously in Columns 1 and 2). That is, the average duration of stay (TOTLOS) in urban-center EDs was 104 minutes longer than the length of stay in periphery region hospitals. Likewise, the average NETLOS in hospitals located in urban centers was 105 minutes longer than that in hospitals located in the periphery. Perceived triage for medical staff was not systematically associated with the location of the hospital.

However, some differences in socio-demographic attributes between patients are apparent across the regions. More specifically, the proportion of men who seek treatment in the ED is slightly higher (55\%) in periphery region hospitals than in central region hospitals (51.4\%), patients in the periphery are younger than patients in the urban center, and they are also less likely to hold an academic degree. Consistent with patterns of spatial segregation in Israeli society, Arabs are three-fold more likely to seek treatment in hospitals located in the periphery than in hospitals located in the urban center.

\section{Multivariate Analysis}

Although the data presented thus far is interesting and quite informative (suggesting that LOS in ED is shorter for Arabs than for Jews and for residents of the periphery than residents of the urban-center), it is unclear from the descriptive statistics whether the shorter length of stay of Arabs can be attributed to ethnic subordination (i.e., differential treatment), spatial location (i.e., peripheral residence), or both. Thus, the analysis that follows estimates a series of regression equations predicting TOTLOS (Table 2) and NETLOS (Table 3), respectively, to arrive at estimates of the net effect of ethnicity and spatial location on length of stay in ED.

In Equation 1 in Table 2, TOTLOS was a function of ethnicity and socio-demographic attributes of patients. Equation 2 includes the spatial location of the hospital (urban center vs. periphery) as an additional predictor of length of stay. For control purposes, Equation 3 introduces several control variables representing the complexity of the medical situation and the set of 18 dummy variables representing the type of the medical problem (not shown in the table). Equation 4 includes a set of dummy variables representing waiting time (triage) for nurses and medical doctors. The regression equations are listed in Table 2. 
Table 2

Coefficients of Regression Equations Predicting Length of Stay (TOTAL LOS) of Patients in Emergency Rooms of the Public Hospitals in Israel*

\begin{tabular}{|c|c|c|c|c|}
\hline Variable & Model 1 & Model 2 & Model 3 & Model 4 \\
\hline Constant & $197.11 * *$ & $160.35 * *$ & $119.61 * *$ & $65.77 *$ \\
\hline Ethnicity $($ Jew =1) & 8.67 & $-19.93^{*}$ & $-19.06 *$ & $-18.08 *$ \\
\hline Gender $($ male $=1)$ & $-16.96^{* *}$ & $-16.43^{*}$ & $-15.07 *$ & $-14.25^{*}$ \\
\hline Education & -5.75 & -11.23 & -10.67 & -9.87 \\
\hline Age (in years) & $1.79 * *$ & $1.65^{* *}$ & $1.58 * *$ & $1.55^{* *}$ \\
\hline Location $($ center $=1)$ & ----- & $98.64 * *$ & $93.85 * *$ & $93.95 * *$ \\
\hline Triage nurse short & ---- & ------ & ------ & .55 \\
\hline Triage nurse mid & ---- & ---- & ---- & .64 \\
\hline Triage nurse long & ---- & ------ & ------ & 23.17 \\
\hline No Triage nurse & & & & Control \\
\hline Triage doctor short & --- & ----- & ---- & 22.00 \\
\hline Triage doctor mid & --- & --- & ---- & $45.2 *$ \\
\hline Triage doctor long & ---- & ----- & ------ & $73.1 * *$ \\
\hline No triage doctor & & & & Control \\
\hline $\begin{array}{l}\text { Medical expert } \\
\text { dummy variables })\end{array}$ & ----- & ------ & See index & Upon request \\
\hline Complexity $($ Urgent $=1)$ & ----- & ----- & $51.99 * *$ & $53.19 * *$ \\
\hline $\mathbf{R}$ square & .023 & .041 & .050 & .056 \\
\hline $\mathbf{N}$ & 9802 & 9802 & 9802 & 9802 \\
\hline
\end{tabular}

$* \mathrm{P} .05, * * \mathrm{P}<.000$

Note: Equation 4 includes a set of 18 dummy variables as a control for the type of medical problem diagnosed (coefficients are not reported and available from the author upon request)

The coefficient for ethnicity in Equation 1 indicates that net of socio-demographic characteristics of patients, the TOTLOS was longer for Jews, by more than 8 minutes $(b=8.67)$, than that for Arabs. However, the 8.67 minutes disparity in TOTLOS was not statistically significant. That is, after taking ethnic differences, age, gender, and education into account, the ethnic disparity in the length of stay in ED is not significant. Equation 1 also suggests that, other things being equal, women tended to spend 16 minutes longer in ED than men $(b=-16.96)$ and that the duration of TOTLOS tended to increase by 1.79 minutes with each year of age. Interestingly, the academic qualification level did not significantly affect the length of stay (in all equations).

Once the hospital location difference is taken into account (in Equation 2), it becomes apparent that TOTLOS of Arab patients was not shorter than that of Jews but actually longer, by almost 20 minutes $(b=-19.9)$ than that of Jewish patients. That is, the shorter TOTLOS of Arabs can be fully attributed to residence in the peripheral regions of the country, where time devoted to patients in emergency units was considerably shorter (by almost 99 minutes) than the TOTLOS in hospitals located in the urban centers $(b=98.64)$. In other words, it is reasonable to conclude from Equation 2 that Arabs are not differentially treated in emergency units due to ethnicity, but rather due to their concentration in the periphery, where TOTLOS for all residents-regardless of ethnicity - is considerably shorter than in the urban-center.

To examine whether and to what extent disparities in TOTLOS are influenced by complexity, urgency, and type of medical problem, a series of variables representing the medical conditions were introduced to Equation 3 for control purposes. The introduction of the variables representing urgency and type of medical problem to Equation 3 do not alter the ethnic disparities 
and the disparities derived from spatial location observed in Equation 2 in any significant way. Furthermore, the disparities remain virtually the same in Equation 4, where perceived waiting time (triage for nurses and medical doctors) was included with the other predictors of TOTLOS. Apparently, triage does not affect the size of the ethnic disparities or spatial disparities in TOTLOS observed in Equations 2 and 3. It should be concluded, therefore, that ethnic disparities in time spent in emergency departments in Israel can be attributed, first and foremost, to the spatial location of the hospital in the country (i.e., where patients spend less time) and not to differential treatment of Jews and Arab patients by the medical staff.

In Table 3, TOTLOS was replaced with NETLOS as the dependent variable to examine whether the net time devoted to medical treatment in the ED differs by ethnicity and by the spatial location of the hospital. Three regression equations were estimated. In Equation 1, NETLOS was predicted as a function of ethnicity plus socio-economic attributes of patients (i.e., age, gender, and education). Equation 2 also includes the spatial location (urban center location versus peripheral location) of the hospital as a predictor of NETLOS. Equation 3 included an additional set of dummy variables, representing the urgency of the problem plus the type of medical problem diagnosed and the urgency of the problem (coefficients of the type of problem are not listed in the table).

\section{Table 3}

Coefficients of Regression Equations predicting "NET LOS" in Emergency Rooms of the Public Hospitals in Israel*

\begin{tabular}{llll}
\hline Variables & Model 1 & Model 2 & Model 3 \\
\hline Constant & $177.70^{* *}$ & $140.92^{* *}$ & $99.75^{* *}$ \\
Ethnicity (Jew =1) & 9.56 & $-19.05^{*}$ & $-18.18^{*}$ \\
Gender (male = 1) & $-16.75^{*}$ & $-16.22^{*}$ & $-14.92^{*}$ \\
Education & -5.45 & -10.93 & -10.38 \\
Age (in years) & $1.78^{* *}$ & $1.64^{* *}$ & $1.58^{* *}$ \\
Location (center=1) & ---- & $98.71^{* *}$ & $93.89^{* *}$ \\
Medical expert (17 dummy & ---- & ---- & See index \\
variables) & & & \\
Complexity (Urgent=1) & ---- & ---- & $52.46^{* *}$ \\
R square & .023 & .041 & .050 \\
\hline N & 9802 & 9802 & 9802 \\
\hline P. $05, * * \mathrm{P}<.000$ & &
\end{tabular}

Note: Equation 3 includes a set of 18 dummy variables as control for the type of medical problem diagnosed (coefficients are not reported and available from the author upon request)

The results presented in Table 3 are highly similar to those shown in Table 2, reaffirming the conclusions reached by the previous analysis. More specifically, the coefficient for ethnicity (b = 9.56) in Equation 1 suggests that the net of socio-demographic attributes of patients, the net time of medical treatment provided to Jewish patients was longer by almost 10 minutes than the time devoted to Arab patients. This disparity, however, was not statistically significant. The data in Equation 1 also show that the time devoted to women was longer than the time devoted to men and that time spent on medical treatment tended to increase with the patient's age. However, the education level was not associated with the net time devoted to medical treatment in ED.

Equation 2 reveals that once the location of the hospital was included as a predictor of NETLOS, the effect of ethnicity on time of medical treatment reverses and became significant (b 
$=-19.05$ ), implying that when considering the over-representation of Arabs in hospitals located in the periphery, the net time devoted for medical treatment of Arabs was actually longer by almost 20 minutes than that devoted to Jews. Indeed, the location of the hospital in the country was the most important determinant of NETLOS. The net time devoted to medical care in ED of hospitals in the urban center was longer, by almost 100 minutes $(b=98.71)$ than the net time devoted to medical care in hospitals located in the periphery. It would seem that due to a lack of resources and shortage of medical staff, hospitals in the periphery devote less time to each ED patient, regardless of ethnicity. These findings remain virtually unchanged when the urgency of the medical problem and the type of the medical problem were controlled for, as in Equation 3. Indeed, the shorter treatment time for Arab patients in the ED can be attributed to residential segregation patterns and not ethnic origin.

\section{Discussion and Conclusion}

The principal objective of the present research was to examine sources explaining disparities in emergency medical treatment in Israeli society between Jews and Arabs (based on ethnicity) and between the urban center and the periphery (based on the spatial location of the hospital). Two indicators of medical service in the emergency department were used: total length of stay (e.g., Asplin et al., 2003; Hoot \& Aronsky, 2008) and net time devoted to medical treatment in the emergency department (e.g., Kyriacou et al., 1999). On average, the length of stay in the ED (whether the total time of the stay or net time devoted to medical care) is considerably shorter for Arabs than for Jews, and for patients in hospitals located in the periphery than for patients in hospitals located in the urban center. However, multivariate analysis reveals that all the ethnic disparities in the length of stay in the emergency department can be attributed to residential segregation patterns.

In fact, when taking into consideration the over-representation of Arabs in small towns and villages in the periphery (where hospitals and medical facilities are scarce, and time devoted to medical care is short), the data reveal that length of stay in emergency units for Arabs was actually longer than that for comparable Jews. In other words, the analysis suggests that the effect of ethnicity on the amount of time devoted to medical care is spurious and can be attributed to patterns of ethnic residential segregation and not to differential treatment by the medical staff (e.g., Feikin et al., 2009; Guagliardo, 2004; LaVeist, 2005; Riley, 2018; Williams \& Collins, 2016). These relations hold even after considering socio-demographic differences and differences in patients' medical condition and type of medical problem of the patients.

The analysis further reveals that the length of medical treatment is influenced, first and foremost, by constraints associated with the condition of hospitals in the periphery, especially the scarcity of hospitals and resources in the periphery (where Arabs tend to reside) (e.g., Lubetzky et al., 2011; Ministry of Health, 2016; Nirel et al., 2000; Shuval, 1990). Specifically, Arabs are more likely to reside in small towns and villages and rural communities in the peripheral regions, where hospitals are scarce, the ratio of patients to physicians is high, and where time for emergency care is shorter by almost 100 minutes compared to emergency departments in the central regions. By way of contrast, Jews are more likely to live in the country's central regions and proximity to the major hospitals, which are equipped with advanced technologies and staffed by a large number of physicians. In other words, superior medical conditions in the hospitals located in urban-center regions enable emergency room physicians to devote extra time to patients for medical treatment, regardless of their ethnicity. The shorter length of time of medical care for Arabs cannot be attributed directly to ethnic origin but indirectly to their spatial segregation. 
To conclude, the findings reveal that Arabs' length of stay for treatment in emergency wards, whether total length of stay or net length of stay, is significantly shorter than Jews' length of stay. The shorter treatment of Arabs in emergency departments, however, cannot be attributed to differential treatment by hospital staff. It is attributed to patterns of spatial segregation in Israel. Arabs in Israel are concentrated in communities located in the peripheral regions of Israel where hospitals are scarce and treatment is short to all patients regardless of their ethnicity. Apparently, residents of the periphery, whether Jews or Arabs, are disadvantaged in terms of access to quality medical services as a result of the differential allocation of medical resources by the state. Indeed, social and state policies and differential allocation of funds and resources across space and populations have become a major source of inequality.

\section{Avenues for Future Research}

The present study relied on length of stay in the emergency department as a measured indicator of quality of healthcare provided to emergency patients. Future research on inequalities in health care services can utilize multiple additional indicators to capture possible differences in quality of care across sub-populations and organizations not only with regard to emergency medicine but also in general medicine. The additional indicators can include both objective measures of health services and subjective evaluations. Furthermore, although the study was carried out within the Israeli context, its theoretical and empirical implications extend far beyond Israel. It is my hope that future studies examine ethnic disparities in health outcomes in other societies to provide a comparative perspective across countries and national healthcare systems and bring deeper understanding of the sources of ethnic health inequality.

\section{Conflict of Interest}

The author declares that the research was conducted in the absence of any commercial or financial relationships that could be construed as a potential conflict of interest.

\section{Acknowledgments}

The author would like to extend her gratitude to Guy Mundlak and Sami Miaari for the helpful comments and suggestions. I would also like to thank the Ministry of Health of Israel for providing the data.

\section{References}

Armstrong, J. (2019). "The Reward was Worth the Sacrifice": How Membership in Black Greek Letter Fraternities (BGLFs) Redefines Black Men's College Experiences. American Journal of Qualitative Research, 3(1), 1-19. https://doi.org/10.29333/ajqr/5808

Asplin, B. R., Magid, D. J., Rhodes, K. V., Solberg, L. I., Lurie, N., \& Camargo Jr, C. A. (2003). A conceptual model of emergency department crowding. Annals of Emergency Medicine, 42(2), 173-180. https://doi.org/10.1067/mem. 2003.302

Baker, F. A., Taher, R., Ganayem, M., Mari, A., \& Kopelman, Y. (2020). Ethnic disparities in colorectal cancer outcomes: A population study from Israel. Ethnicity \& Health, 1-11. https://doi.org/10.1080/13557858.2020.1795630 
Blanchard, J. C., Haywood, Y. C., \& Scott, C. (2003). Racial and ethnic disparities in health: An emergency medicine perspective. Academic Emergency Medicine, 10(11), 1289-1293. https://doi.org/10.1197/S1069-6563(03)00501-3

Bowen, S. (2001). Language barriers in access to health care. Health Canada.

Carter, R. T., Lau, M. Y., Johnson, V., \& Kirkinis, K. (2017). Racial discrimination and health outcomes among racial/ethnic minorities: A meta-analytic review. Journal of Multicultural Counseling \& Development, 45(4), 232-259. https://doi.org/10.1002/jmcd.12076

Chaou, C. H., Chen, H. H., Chang, S. H., Tang, P., Pan, S. L., Yen, A. M. F., \& Chiu, T. F. (2017). Predicting length of stay among patients discharged from the emergency departmentusing an accelerated failure time model. PloS one, 12(1), e0165756. https://doi.org/10.1371/journal.pone.0165756

Chapman, E. N., Kaatz, A., \& Carnes, M. (2013). Physicians and implicit bias: How doctors may unwittingly perpetuate health care disparities. Journal of General Internal Medicine, 28, 1504-1510. https://doi.org/10.1007/s11606-013-2441-1

Daoud, N., Soskolne, V., Mindell, J. S., Roth, M. A., \& Manor, O. (2018). Ethnic inequalities in health between Arabs and Jews in Israel: The relative contribution of individual-level factors and the living environment. International Journal of Public Health, 63, 313-323. https://doi.org/10.1007/s00038-017-1065-3

Dehon, E., Weiss, N., Jones, J., Faulconer, W., Hinton, E., \& Sterling, S. (2017). A systematic review of the impact of physician implicit racial bias on clinical decision making. Academic Emergency Medicine, 24(8), 895-904. https://doi.org/10.1111/acem.13214

Feikin, D. R., Nguyen, L. M., Adazu, K., Ombok, M., Audi, A., Slutsker, L., \& Lindblade, K. A. (2009). The impact of distance of residence from a peripheral health facility on pediatric health utilisation in rural western Kenya. Tropical Medicine \& International Health, 14(1), 54-61. https://doi.org/10.1111/j.1365-3156.2008.02193.x

Fiscella, K., Franks, P., Gold, M. R., \& Clancy, C. M. (2000). Inequality in quality: Addressing socioeconomic, racial, and ethnic disparities in health care. JAMA, 283(19), 2579-2584. https://doi.org/10.1001/jama.283.19.2579

Flores, G. (2006). Language barriers to health care in the United States. New England Journal of Medicine, 355(3), 229-231. https://doi.org/10.1056/NEJMp058316

Guagliardo, M. F. (2004). Spatial accessibility of primary care: Concepts, methods and challenges. International Journal of Health Geographics,3(1), 3. https://doi.org/10.1186/1476-072X-3-3

Harris, R. B., Stanley, J., \& Cormack, D. M. (2018). Racism and health in New Zealand: Prevalence over time and associations between recent experience of racism and health and wellbeing measures using national survey data. PloS one, 13(5), e0196476.

Hatab, N., \& Kagya, S. (2011). Health Inequalities between Arabs and Jews in Israel. The Israel Democracy Institute. (Hebrew).

Herring, A., Wilper, A., Himmelstein, D. U., Woolhandler, S., Espinola, J. A., Brown, D. F., \& Camargo Jr., C. A. (2009). Increasing length of stay among adult visits to US emergency departments, 2001-2005. Academic Emergency Medicine, 16(7), 609-616. https://doi.org/10.1111/j.1553-2712.2009.00428.x

Hoot, N. R., \& Aronsky, D. (2008). Systematic review of emergency department crowding: Causes, effects, and solutions. Annals of Emergency Medicine, 52(2), 126-136. https://doi.org/10. 1016/j.annemergmed.2008.03.014

Israel Ministry of Health. (2010). Health inequalities and ways to cope with it. Israel Ministry of Health. 
James, W. Y. (2019). Imprint of Racism: White Adult Males' Transformational Experience from Racial Antipathy to Racial Reconciliation. American Journal of Qualitative Research, 3(1), 93-116. https://doi.org/10.29333/ajqr/5813

Ku, L., \& Matani, S. (2001). Left out: Immigrants' access to health care and insurance. Health Affairs, 20(1), 247-256. https://doi.org/10.1377/hlthaff.20.1.247

Kyriacou, D. N., Ricketts, V., Dyne, P. L., McCollough, M. D., \& Talan, D. A. (1999). A 5-year time study analysis of emergency department patient care efficiency. Annals of Emergency Medicine, 34(3), 326-335. https://doi.org/10. 1016/S0196-0644(99)70126-5

LaVeist, T. A. (2005). Disentangling race and socio-economic status: A key to understanding health inequalities. Journal of Urban Health, 82(3), iii26-iii34. https://doi.org/10.1093/ jurban/jti061

Lewin-Epstein, N., \& Semyonov, M. (1993). The Arab minority in Israel's economy: Patterns of ethnic inequality. Routledge.

Lubetzky, H., Friger, M., Warshawsky-Livne, L., \& Shvarts, S. (2011). Distance and socioeconomic status as a health service predictor on the periphery in the southern region of Israel. Health Policy, 100(2-3), 310-316. https://doi.org/10.1016/j.healthpol.2010.09.006

Mackenbach, J. P., Stirbu, I., Roskam, A. J. R., Schaap, M. M., Menvielle, G., Leinsalu, M., \& Kunst, A. E. (2008). Socio-economic inequalities in health in 22 European countries. New England Journal of Medicine, 358(23), 2468-2481. https://www.nejm.org/doi/full/10.1056/nejmsa0707519

Manor, O., Eisenbach, Z., Israeli, A., \& Friedlander, Y. (2000). Mortality differentials among women: The Israel longitudinal mortality study. Social Science \& Medicine, 51(8), 11751188. https://doi.org/10.1016/S0277-9536(00)00024-1

Meuter, R. F., Gallois, C., Segalowitz, N. S., Ryder, A. G., \& Hocking, J. (2015). Overcoming language barriers in healthcare: A protocol for investigating safe and effective communication when patients or clinicians use a second language. BMC Health Services Research, 15(1), 371. https://doi.org/10.1186/s12913-015-1024-8

Ministry of Health, State of Israel. (2016). Inequality report. (Hebrew).

Miro, O., Antonio, M. T., Jimenez, S., De, A. D., Sanchez, M., Borras, A., \& Millá, J. (1999). Decreased health care quality associated with emergency department overcrowding. European Journal of Emergency Medicine, 6(2), 105-107.

Monheit, A. C., \& Vistnes, J. P. (2000). Race/ethnicity and health insurance status: 1987 and 1996. Medical Care Research and Review, 57(1_suppl), 11-35.

Morrill, R. L., Earickson, R. J., \& Rees, P. (1970). Factors influencing distances traveled to hospitals. Economic Geography, 46(2), 161-171. https://doi.org/10.2307/142624

Moskop, J. C., Sklar, D. P., Geiderman, J. M., Schears, R. M., \& Bookman, K. J. (2009). Emergency department crowding, part 1 - Concept, causes, and moral consequences. Annals of Emergency Medicine, 53(5), 605-611. https://doi.org/10. 1016/j.annemergmed.2008.09.019

Mowery, N. T., Dougherty, S. D., Hildreth, A. N., Holmes, J. H., Chang, M. C., Martin, R. S., Hoth, J. J., Meredith, J. W., \& Miller, P. R. (2011). Emergency department length of stay is an independent predictor of hospital mortality in trauma activation patients. The Journal of Trauma, 70(6), 1317-1325.

Nazroo, J. Y. (2003). The structuring of ethnic inequalities in health: economic position, racial discrimination, and racism. American Journal of Public Health,93(2), 277-284. https://doi.org/10.2105/AJPH.93.2.277 
Nirel, N., Pilpel, D., Rosen, B., Zmora, I., Greenstein, M., \& Zalcberg, S. (2000). The accessibility and availability of health services in the south of Israel: Has the gap between the south and other regions in Israel been reduced in the wake of the National Health Insurance Law. The Myers-JDC-Brookdale Institute.

Padela, A. I., \& Punekar, I. R. (2009). Emergency medical practice: Advancing cultural competence and reducing health care disparities. Academic Emergency Medicine, 16(1), 69-75. https://doi.org/10.1111/j.1553-2712.2008.00305.x

Pines, J. M., Localio, A.R., \& Hollander, J. E. (2009). Racial disparities in emergency department length of stay for admitted patients in the United States. Academic Emergency Medicine, 16(5), 403-410. https://doi.org/10.1111/j.1553-2712.2009.00381.x

Richardson, L. D., Babcock Irvin, C., \& Tamayo-Sarver, J. H. (2003). Racial and ethnic disparities in the clinical practice of emergency medicine. Academic Emergency Medicine, 10(11), 1184-1188. https://doi.org/10.1197/ S1069-6563(03)00487-1

Riley, A. R. (2018). Neighborhood disadvantage, residential segregation, and beyond-Lessons for studying structural racism and health. Journal of Racial \& Ethnic Health Disparities, 5(2), 357-365. https://doi.org/10.1007/s40615-017-0378-5

Saabneh, A. M. (2015). Arab-Jewish gap in life expectancy in Israel. The European Journal of Public Health, 26(3), 433-438. https://doi.org/10.1093/eurpub/ckv211

Semyonov, M., Lewin-Epstein, N., \& Bridges, W. P. (2011). Explaining racial disparities in access to employment benefits. Ethnic \& Racial Studies, 34(12), 2069-2095. https://doi.org/10.1080/01419871003687552

Semyonov, M., Lewin-Epstein, N., \& Maskileyson, D. (2013). Where wealth matters more for health: The wealth-health gradient in 16 countries. Social Science \& Medicine, 81, 10-17. https://doi.org/10.1016/j.socscimed.2013.01.010

Shuval, J. T. (1990). Health in Israel: Patterns of equality and inequality. Social Science \& Medicine, 31(3), 291-303. https://doi.org/10.1016/0277-9536(90)90276-X

Sprivulis, P. C., Da Silva, J. A., Jacobs, I. G., Jelinek, G. A., \& Frazer, A. R. (2006). The association between hospital overcrowding and mortality among patients admitted via Western Australian emergency departments. Medical Journal of Australia, 184(5), 208212. https://doi.org/10.5694/j.1326-5377.2006.tb00203.x

Thomas, J. W., Guire, K. E., \& Horvat, G. G. (1997). Is patient length of stay related to quality of care? Journal of Healthcare Management, 42(4), 489-507.

Williams, D. R., \& Collins, C. (1995). US socio-economic and racial differences in health: Patterns and explanations. Annual Review of Sociology, 21(1), 349-386. http://www.jstor.org/ stable/ 2083415

Williams, D. R., \& Collins, C. (2016). Racial residential segregation: A fundamental cause of racial health disparities. Public Health Reports, 116(5), 404-416. https://doi.org/10.1093/phr/116.5.404

Williams, D. R., \& Sternthal, M. (2010). Understanding racial-ethnic disparities in health: Sociological contributions. Journal of Health \& Social Behavior, 51(1_suppl), S15-S27. https://doi.org/10.1177 /0022146510383838

Zuvekas, S. H., \& Taliaferro, G. S. (2003). Pathways to access: Health insurance, the health care delivery system, and racial/ethnic disparities, 1996-1999. Health Affairs, 22(2), 139-153. https://doi.org/10.1377/hlthaff.22.2.139 


\section{Notes on Contributor}

Keren Semyonov Tal is a $\mathrm{PhD}$ candidate in the Department of Labor Studies at Tel Aviv University. Prior to starting her graduate studies, she practiced law. Her research interests lie in the areas of health rights and social inequality; her dissertation focuses on provision of dignity and respect to patients in hospitals. Articles dealing with patients' satisfaction were recently published in Health Policy Open and Journal of Patient Experience.

\section{Appendix}

\section{Table A}

Variables used in the Analysis, Definitions, and Mean (s.d.) or Percentage

\begin{tabular}{|c|c|c|}
\hline Variable name & Nominal definition & $\begin{array}{l}\text { Optional } \\
\text { definition }\end{array}$ \\
\hline TOTAL LOS & Duration of stay in the emergency ward & In minutes \\
\hline NET LOS & LOS - (SUM triage doctor + triage nurse $)$ & In minutes \\
\hline Education & 2 categories of educational attainment (\%) & $\begin{array}{l}\text { Non-academic } \\
\text { Academic }=1\end{array}$ \\
\hline Gender & Gender of respondent (\%) & Female \\
\hline & & Male $=1$ \\
\hline Ethnicity & $\begin{array}{l}\text { Ethnic origin of respondent defined by } \\
\text { language used }(\%)\end{array}$ & $\begin{array}{l}\text { Arabic } \\
\text { Hebrew+ Russian= } \\
1\end{array}$ \\
\hline
\end{tabular}

\begin{tabular}{|c|c|c|c|}
\hline Age & In years & & $\begin{array}{l}36.31 \\
(26.68)\end{array}$ \\
\hline \multirow[t]{2}{*}{ Location } & \multirow[t]{2}{*}{ Regional location of the hospital (\%) } & Peripheral & 31.7 \\
\hline & & Central $=1$ & 68.3 \\
\hline $\begin{array}{c}\text { Complexity of } \\
\text { hospitalization }\end{array}$ & & $\begin{array}{l}\text { Regular ED } \\
\text { Urgent } E D=1\end{array}$ & $\begin{array}{l}5.2 \\
94.8\end{array}$ \\
\hline \multirow{4}{*}{$\begin{array}{l}\text { TRIAGE } \\
\text { nurses }\end{array}$} & \multirow{4}{*}{$\begin{array}{l}\text { Time of waiting before medical treatment } \\
\text { reported by patients }(\%)\end{array}$} & No Wait $(0 \mathrm{~min})$ & 5.9 \\
\hline & & Short wait (5 min) & 36.5 \\
\hline & & Reasonable wait & 37.6 \\
\hline & & $\begin{array}{l}(10 \mathrm{~min}) \\
\text { Long wait (15 min) }\end{array}$ & 19.9 \\
\hline \multirow{4}{*}{$\begin{array}{l}\text { TRIAGE } \\
\text { doctors }\end{array}$} & \multirow{4}{*}{$\begin{array}{l}\text { Time of waiting before medical treatment } \\
\text { reported by patients }(\%)\end{array}$} & No Wait $(0 \mathrm{~min})$ & 3 \\
\hline & & Short wait (5 min) & 25.3 \\
\hline & & Reasonable wait & 38.3 \\
\hline & & $\begin{array}{l}(10 \mathrm{~min}) \\
\text { Long wait (15 min }\end{array}$ & 33.4 \\
\hline Triage nurses & $X$ in minutes & & $8.57(4.2)$ \\
\hline \multirow[t]{2}{*}{ Triage doctor } & \multirow[t]{2}{*}{$X$ in minutes } & & $10.11(4.19)$ \\
\hline & & & $\mathrm{N}=9803$ \\
\hline
\end{tabular}


Table B

Percent Distribution of the Type of Medical Problem Diagnosed Among Patients in the Emergency Unit of Public Hospitals in Israel by Ethnicity/Location \%

\begin{tabular}{lccccc}
\hline Medical problem & Arab & Jew & Periphery & Center & All \\
\hline Surgeon=1 & 18.2 & 20.1 & 19.1 & 19.9 & 19.6 \\
Orthopedist=2 & 33.2 & 30.7 & 33.1 & 30 & 31.0 \\
Neurologist=3 & 3.5 & 6.2 & 4.4 & 6.5 & 5.8 \\
Otolaryngology=4 & 7.6 & 7.7 & 8.9 & 7 & 7.6 \\
Eyes=5 & 3.7 & 6.4 & 5.6 & 6.1 & 5.9 \\
Plastics=6 & .9 & 1.3 & 1.5 & 1.2 & 1.3 \\
Urologist=7 & 3.8 & 3.9 & 3.7 & 4 & 3.9 \\
Cardiologist=8 & 4.9 & 4.9 & 4.1 & 5.4 & 5.0 \\
Oncologist=9 & .9 & .6 & .3 & .7 & .6 \\
Skin=10 & 1.2 & 1 & 1.3 & 1 & 1.1 \\
Neurosurgeon=11 & .6 & .7 & .3 & .8 & .68 \\
Others=12 & 6.5 & 5.8 & 5.5 & 6.3 & 6.0 \\
Pediatrician=13 & 6.9 & 3.1 & 4.4 & 3.4 & 3.7 \\
Nephrologist=14 & .1 & .4 & .5 & .3 & .4 \\
Internal medicine=15 & 6 & 3.9 & 5.7 & 3.6 & 4.3 \\
Pulmonary physician=16 & .4 & .7 & .2 & .9 & .6 \\
Gastroenterologist= 17 & .6 & .6 & .5 & .6 & .6 \\
Gynecologist= 18 & .9 & 1.9 & .8 & 2.2 & 1.8 \\
\hline
\end{tabular}

